\title{
Grating Lobes Suppression in Frequency Selective Surfaces Using Electromagnetic Band Gap with Square Holes
}

\author{
Tiago dos Santos Bezerra ${ }^{1}$ (D), Antonio Luiz Pereira de Siqueira Campos $^{1}$ (D), Ruann Victor de \\ Andrade Lira ${ }^{1}$ (D), Alfredo Gomes Neto ${ }^{2}$ (D) \\ ${ }^{1}$ Federal University of Rio Grande do Norte, Av. Senador Salgado Filho, 3000, Natal, RN, Brazil, \\ tiagosabezerra@gmail.com, alpscampos@gmail.com,ruannvictor@gmail.com \\ ${ }^{2}$ Federal Institute of Paraiba, Av Primeiro de Maio, 720, Jaguaribe, João Pessoa, PB, Brazil, \\ alfredogomesjpa@gmail.com
}

\begin{abstract}
In this paper, the authors present a proposal for an application of electromagnetic bandgap (EBG), for suppression of grating lobes, in frequency selective surfaces (FSS), not yet studied in the literature. An alternative type of EBG with square holes was proposed to reduce the computational effort in simulations. The study consists of an application of a rectangular periodic array of cylindrical and square holes in FSS dielectric substrate to create rejection bands and suppress specific resonant frequency modes. We built four prototypes and compared measured results with simulated results obtained with ANSYS HFSS. Simulations and measurements show suppression levels up to $6 \mathrm{~dB}$. A good agreement between the results is observed. The FSS with EBG with square holes allows a simulation time $70 \%$ lower than FSS with EBG with cylindrical holes.
\end{abstract}

Index Terms - FSS, EBG, Suppression, Grating lobes.

\section{INTRODUCTION}

Planar microstrip structures have been used in several applications, for wireless communication systems, due to the low cost of manufacture, reduced weight and dimensions, and ease of manufacture on a large scale [1], [2]. Among some of these applications, antennas, filters, and frequency selective surfaces (FSS) can be mentioned. In some cases, these structures may present radiation of spurious modes [3], which can cause interference in antennas of other communication systems [4], or degrade the level of rejection above the passband, in the case of microstrip filters [5] and on frequency selective surfaces (FSS), spurious modes can significantly degrade the frequency response in the passband [6].

Several techniques have been used to suppress spurious modes, such as the use of defects in the ground plane (Defected ground structure - DGS) of planar antennas [7], [8], or the use of metamaterials in microstrip filters [9], [10] and the use of electromagnetic bandgap (EBG) in planar antennas [11], [12]. However, it is not known to the authors the use of these techniques for suppression or reduction grating lobes in FSS.

Most studies, found in literature, address EBG with cylindrical holes. Few studies using EBG 
substrates with non-cylindrical holes are reported [13], [14]. Thus, it is important to advance in the study of spurious suppression in planar structures, researching the application of EBG in FSS, aiming at the reduction or suppression of grating lobes. Also, the manufacture and application of EBG dielectrics with non-cylindrical holes are necessary, as it can reduce computational effort.

In this paper, a 3D printer was used to produce EBG dielectric substrates, with square holes, to be applied in FSS and to suppress grating lobes. An EBG dielectric with cylindrical holes was also manufactured, for comparison purposes, in terms of computational effort and manufacturing difficulty. Numerical and experimental results are presented.

\section{Proposed Structures}

EBGs are periodic or quasi-periodic arrays inserted in conductive or dielectric materials. These arrays introduce rejection bands that prohibit the propagation of electromagnetic waves in certain frequencies [15]. In this work, we will analyze two types of substrates with EBG, one with a periodic array of cylindrical holes and the other with a periodic array of square holes. The idea is to suppress grating lobes and to compare the computational effort of the simulations made for the two types of arrays.

Fig. 1(a) illustrates the periodic EBG array with cylindrical holes in the dielectric, as well as their physical dimensions, which are the distance between the centers of the air holes, $\Lambda$ (pitch), and the diameter of the holes, $d$. Fig. 1(b) illustrates the periodic EBG array with square holes, as well as their physical dimensions, which are the distance between the centers of the air holes, $\Lambda$ (pitch), and the width of the holes, $W_{a r}$. In the case of the cylindrical hole array, we used a Technodrill milling machine model Sigma 600 to perforate the FR-4 dielectric. In the case of the square hole array, a 3D printer was used to manufacture the dielectric. The main motivation to construct the dielectric with square holes is that the fabrication process using $3 \mathrm{D}$ printer is accurate enough and low-cost, and the computational effort of the simulation is lower when compared with EBG with cylindrical holes.

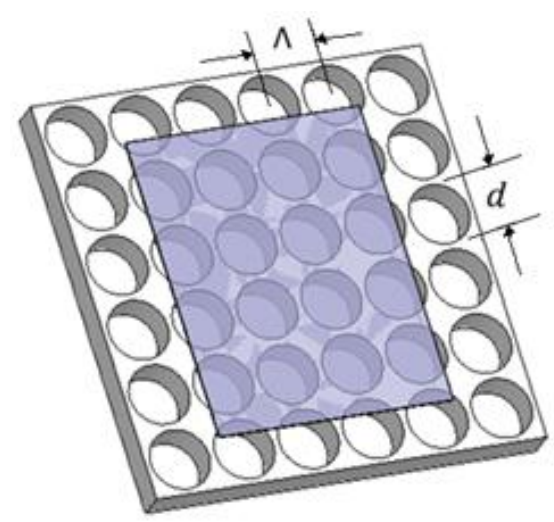

(a)

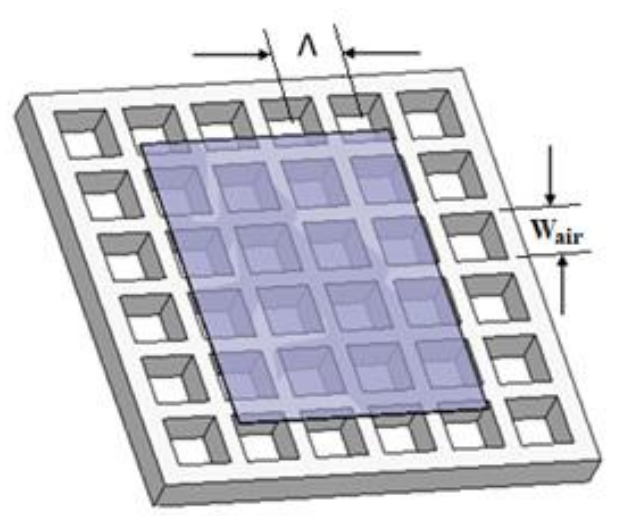

(b)

Fig. 1. EBG array with: (a) cylindrical holes and (b) square holes.

A good starting point for the dimensions of the EBG with cylindrical holes, pitch, and diameter, is provided in [14], which is $\Lambda=0.12 \lambda$ and $d=0.1 \lambda$, where $\lambda$ is the wavelength of the grating lobe 
frequency to be suppressed. For the case of square holes, the area of the hole must be equal to the area of the cylindrical hole, maintaining the areas of the holes of the EBG array and, consequently, the desired rejection band. Thus, $W_{a r}$ should be approximately $0.89 d$, where $d$ is the diameter of the cylindrical holes.

Fig. 2(a) illustrates the FSS unit cell, considered for the study proposed in [16]. The geometry is a rectangular patch with dimensions $L=15 \mathrm{~mm}, W=12 \mathrm{~mm}$, and the periodicity of the unit cell is $P=$ $20 \mathrm{~mm}$. The metallic portion of the unit cell is gray color. A FR-4 dielectric substrate (fiberglass), with $1.6 \mathrm{~mm}$ of thickness, the relative permittivity of 4.4 and a loss tangent of 0.02 was used. Fig. 2(b) illustrates the cell of the FSS unit considered with the EBG inserted in the FR-4 dielectric. The unit cell of the FSS is the same as Fig. 2(a). The air holes have a diameter $d=3 \mathrm{~mm}$, with a spacing between the holes (pitch) of $3.3 \mathrm{~mm}$. A periodic matrix of $6 \times 6$ holes was inserted into each unit cell, resulting in 36 holes per cell. The dielectric used was the FR-4. Fig. 2(c) illustrates the FSS unit cell considered on a substrate with EBG of square holes. The unit cell of the FSS is the same of Fig. 2(a). The material used for the substrates was the acrylonitrile-butadiene-styrene (ABS). ABS material is the most used polymer in 3D printing due to its low cost, ease of printing, lightweight and good rigidity with relative flexibility. The printer used was the VOID1 model. The print quality was 0.05 $\mathrm{mm}$ per height of the deposited layer. 3D printing was used to enable the production of square holes. The spacing between the holes (pitch) was kept as $3.3 \mathrm{~mm}$ and, for the square hole to have approximately the same area as the cylindrical hole, the side should be equal to $2.67 \mathrm{~mm}$, but due to the precision offered by the printer, the final value was equal to $2.5 \mathrm{~mm}$. The dielectric has $1.6 \mathrm{~mm}$ of thickness and a relative permittivity of 3.3 .

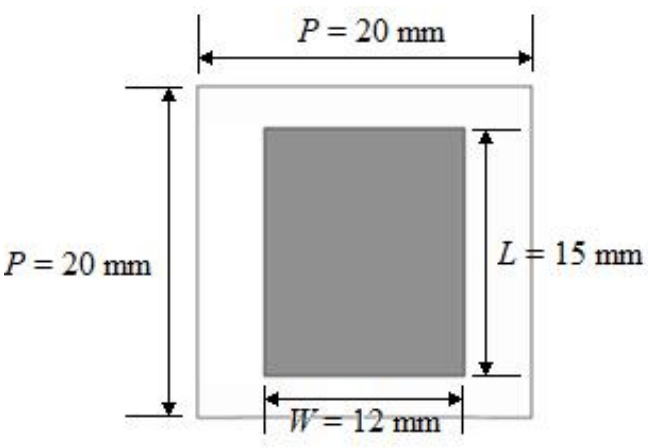

(a)

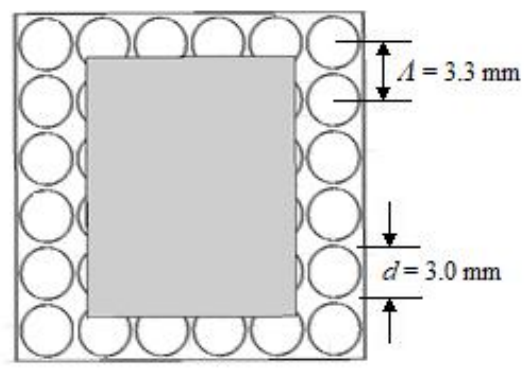

(b)

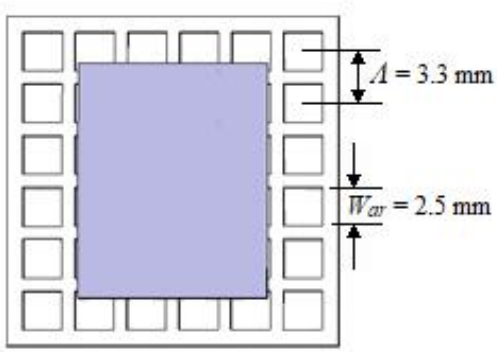

(c)

Fig. 2. FSS unit cell: (a) original cell, (b) with EBG with cylindrical holes and (c) with EBG with square holes. Brazilian Microwave and Optoelectronics Society-SBMO received 4 May 2020; for review 7 May 2020; accepted 1 Oct 2020 
To show how the mechanism to suppression of higher order modes by the EBG works, we must to characterize the behavior of the wave as it propagates through the structure. This is made with the dispersion diagram, also named is Brillouin diagram. The computational domain and the boundary setup for extracting the dispersion diagram for the EBG unit cell were shown in [17] and [18]. The full-wave numerical simulator Ansof HFSS is used to analyze the 2D propagating and nonpropagating modes on the Brillouin diagram. We obtained the diagrams for the cells shown in Fig. 2(b) and (c).

We can see the dispersion diagram generated for the EBG of Fig. 2(b) in Fig. 3. We obtain the diagram for the first six propagating modes extracted numerically for this structure. It shows that the bandgap is located between $11.85 \mathrm{GHz}$ and $13.85 \mathrm{GHz}$ and it shows that there will be no resonance at this band for the FSS. In Fig. 4, we can see the dispersion diagram generated for the EBG of Fig. 2(c). In this case, the bandgap is located between $11.00 \mathrm{GHz}$ and $13.90 \mathrm{GHz}$, a little bit larger that the bandgap obtained for the EBG of the Fig. 2(b). So, as the diagrams show we have bandgaps in the range of interest, that can suppress the higher order modes.

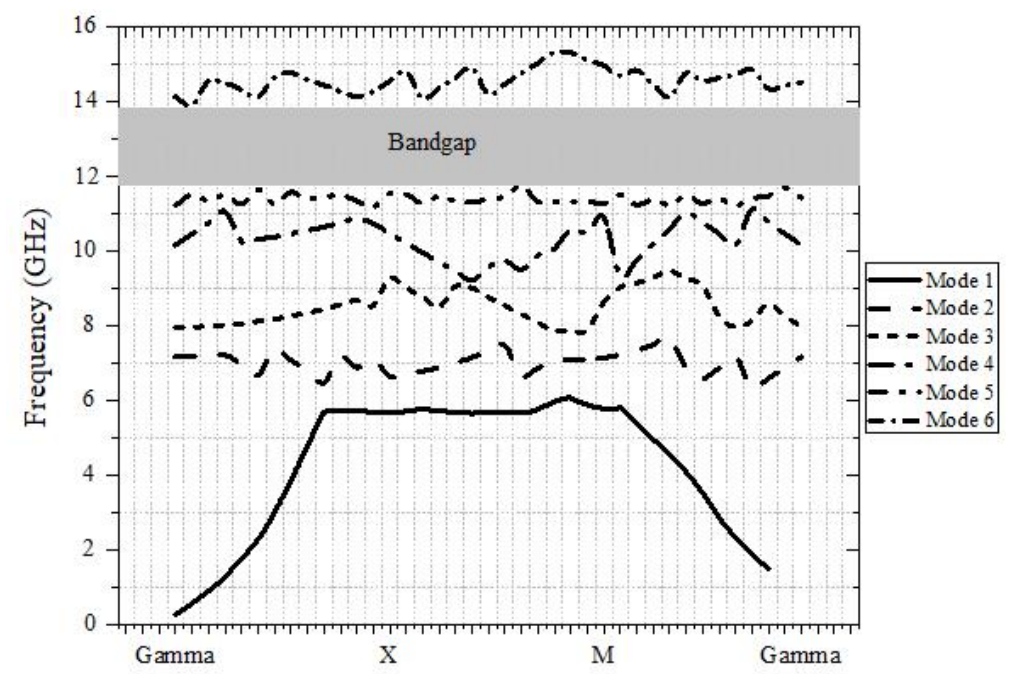

Fig. 3. Dispersion diagram of the planar EBG structure shown in Fig. 2(b).

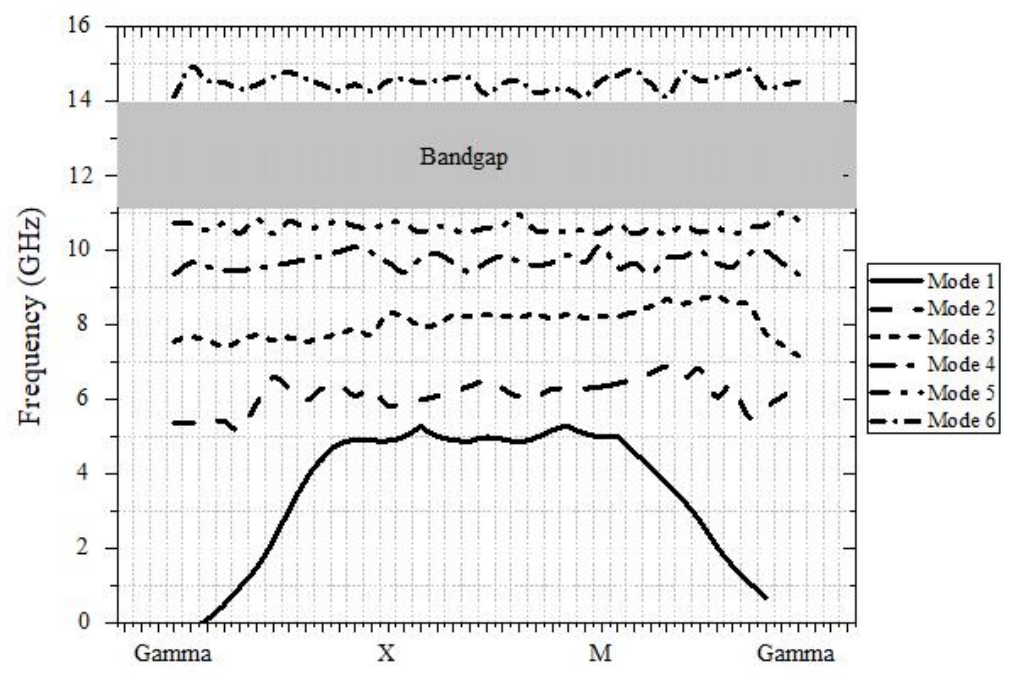

Fig. 4. Dispersion diagram of the planar EBG structure shown in Fig. 2(c). 
Grating lobes occur when the lattice size of the FSS becomes electrically large [19]. The largest lattice size to avoid grating lobes obey the same rule that one for conventional array antenna. The rule is that the lattice size should be less than $\lambda$. This rule limits the possible dimensions in an FSS design. Our proposition changes this and we can eliminate the grating lobe with an EBG. This includes one more possibility to eliminate grating lobe and also contributes to the research area in question.

Fig. 5 illustrates the transmission coefficient of the structures in Fig. 2. The original FSS has a resonant frequency at $9.24 \mathrm{GHz}$ and a grating lobe at $13.02 \mathrm{GHz}$. When we insert the EBG with cylindrical holes, the resonant frequency changes from $9.24 \mathrm{GHz}$ to $10.07 \mathrm{GHz}$. This was expected due to the air holes that reduce the effective permittivity $\left(\varepsilon_{e f f}\right)$ of the FSS substrate, because of this the resonance frequency increases. We can also see that the grating lobe has been attenuated by $6 \mathrm{~dB}$, from $-11 \mathrm{~dB}$ to $-5 \mathrm{~dB}$. For the case of FSS with a substrate with EBG of square holes made in the 3D printer, we can see that the resonance frequency increases to $11.86 \mathrm{GHz}$, and the grating lobe was also attenuated by $6 \mathrm{~dB}$, as well. In this case, the relative permittivity is 3.3 and the effective permittivity $\left(\varepsilon_{e f f}\right)$ of the FSS substrate is less than for the EBG with cylindrical holes, and the resonance frequency is greater.

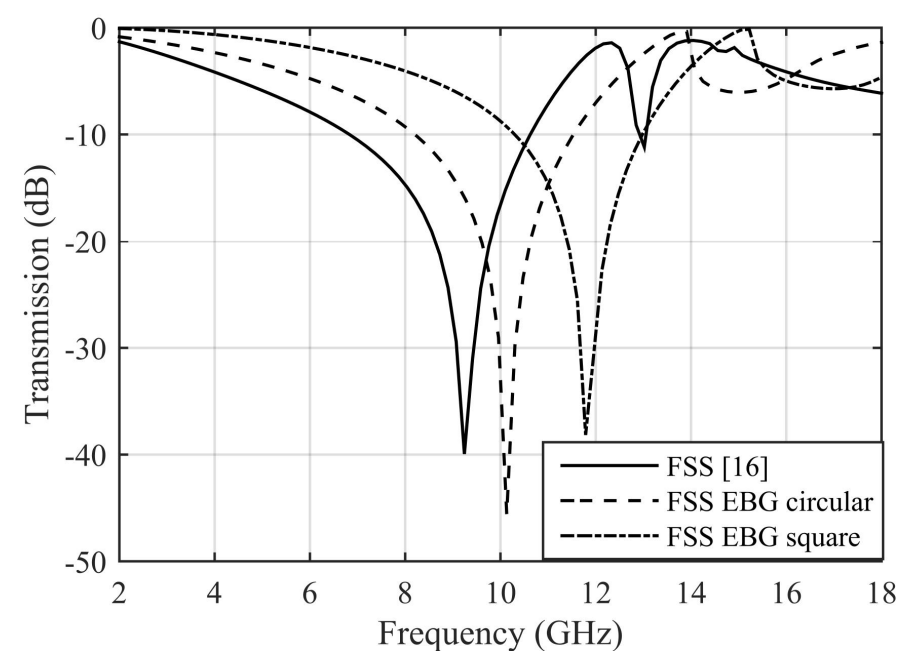

Fig. 5. Transmission coefficient for the FSS proposed in [16] without EBG, with EBG with cylindrical holes and with EBG with square holes.

To return the resonance to the original frequency, $9.24 \mathrm{GHz}$, we can resize the dimensions of the rectangular patch, causing the resonance frequency to return to the desired value. So, for EBG with square holes, we resized the patch of the unit cell. Its length changed to $18.5 \mathrm{~mm}$. Fig. 6 illustrate a comparison between the transmission response of the original FSS and the FSS with EBG with square holes. As we can see, the resonant frequency is $10.22 \mathrm{GHz}$, near to $9.24 \mathrm{GHz}$ and the grating lobe was suppressed. 


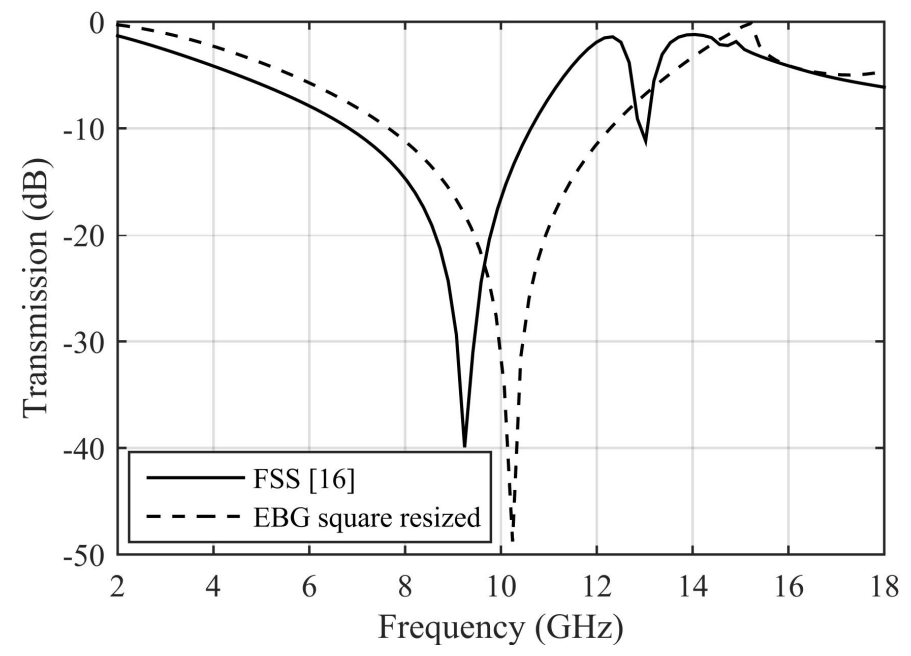

Fig. 6. Transmission coefficient for the FSS proposed in [16] without EBG and for the FSS with EBG with square holes and $L=18.5 \mathrm{~mm}$.

\section{COMputational Performance}

In this section, we present one of the advantages of using a different hole geometry than conventional cylindrical holes. Fig. 7 illustrates the simulation mesh of the three unit cells considered in this study. These meshes were obtained using the ANSYS HFSS software. It is observed that the mesh with less subdivision (triangles, in this case) is the FSS of [16], without EBG, followed by the FSS with EBG with square holes. The mesh of the FSS with cylindrical holes is the most detailed of all. How much more is the number of subdivisions, much more will be the computational effort of the simulation. This is an important point, especially if an optimization tool is used.

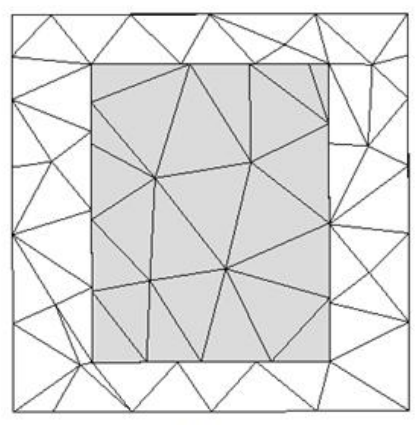

(a)

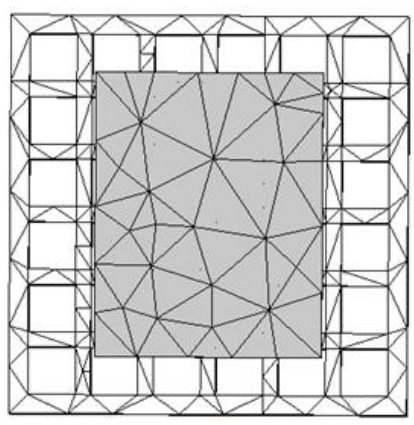

(b)

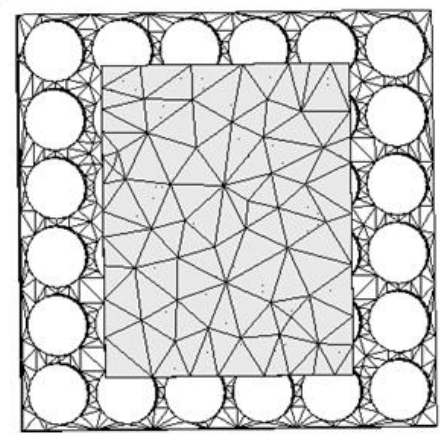

(c)

Fig. 7. FSS simulation mesh: (a) FSS without holes, (b) FSS with EBG with square holes and (c) FSS with EBG with cylindrical holes. 
All simulations were made using the range of frequency of $2 \mathrm{GHz}$ to $18 \mathrm{GHz}$, in discrete mode and with 100 points. This greater detail of the mesh leads to longer simulation time. To analyze the simulation time, the same computational conditions were applied to all FSS. The FSS simulation time with EBG with cylindrical holes was 4 hours and 53 minutes. On the other hand, it took 1 hour and 26 minutes to simulate the FSS with EBG with square holes and for the FSS without EBG the time simulation was 47 minutes. Table 1 listed the main mesh variables and as we can see, the EBG with cylindrical holes has more complex parameters.

TABLE I. MESh STATISTICS

\begin{tabular}{cccc}
\hline \multirow{2}{*}{ Statistic parameter } & \multicolumn{3}{c}{ Structure } \\
\cline { 2 - 4 } & FSS without EBG & EBG with square holes & EBG with cylindrical holes \\
\hline Total number of mesh element & 2385 & 4999 & 19952 \\
Number of Tetrahedrons & 1902 & 3686 & 15117 \\
Min Edge Length & 1.77328 & 0.57567 & 0.445852 \\
Max Edge Length & 8.87647 & 8.45802 & 5.43731 \\
RMS Edge Length & 5.38779 & 4.38524 & 2.63568 \\
Mean Tetrahedron (vol) & 5.12198 & 0.0013979 & 0.00110174 \\
Std Devn Tetrahedron (vol) & 4.78665 & 2.76183 & 0.701916 \\
\hline
\end{tabular}

\section{RESUlTS AND DisCUSSIONS}

For validation purposes of the proposed technique in this work, we built and measured four FSS prototypes. A prototype of the first analyzed FSS without EBG was manufactured and three FSS prototypes with EBG were manufactured. We built two prototypes with an FR-4 substrate with relative permittivity of 4.4 , loss tangent of 0.02 , a thickness of $1.6 \mathrm{~mm}$, and dimensions of $23 \mathrm{~mm} \times$ $23 \mathrm{~mm}$. In the case of FSS with EBG, the holes were made with a prototype Tecnodrill ${ }^{\circledR}$ model Sigma 600. After making the holes, the patches were manufacture manually, with a $50 \mu \mathrm{m}$ conductive adhesive copper foil tape. Fig. 8 illustrates the process of making the cylindrical holes.

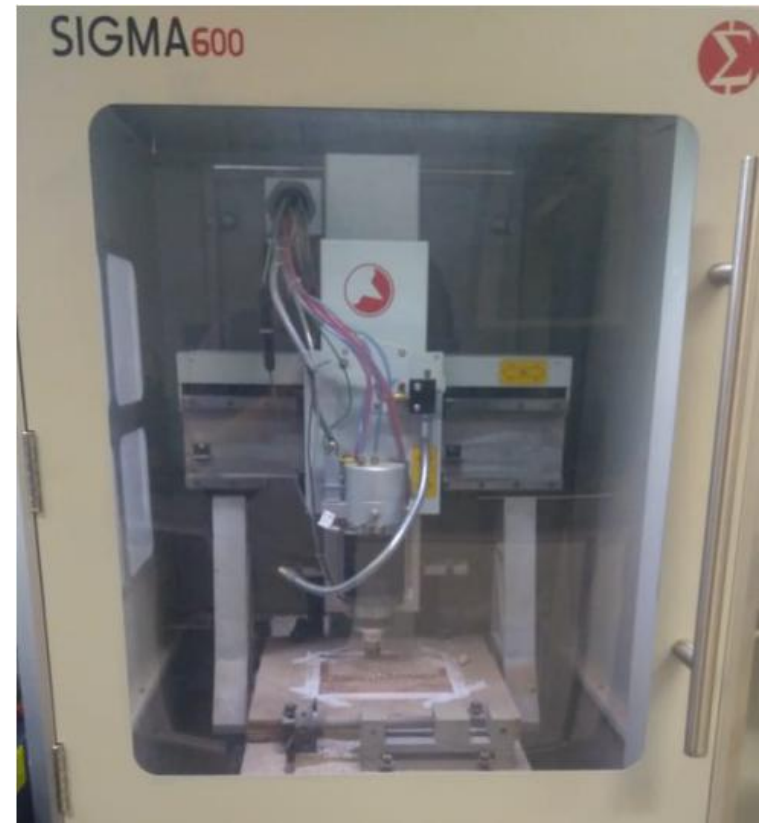

Fig. 8. Making holes in a prototyper.

Brazilian Microwave and Optoelectronics Society-SBMO received 4 May 2020; for review 7 May 2020; accepted 1 Oct 2020 
The other two prototypes were made in a fused deposition modeling (FDM) 3D printer. The FDM process was used for the construction of a substrate with square hole EBG. In the process, the thermoplastic filament selected was ABS, widely used in the industry due to its low cost, as it is a light material and easy to model in addition to having good resistance to impact, traction, and abrasion. The construction of the structures was carried out in the 3D printing machine of model A8 of the manufacturer Anet (with a precision of up to $0.2 \mathrm{~mm}$ depending on the extrusion nozzle used) using an extrusion nozzle of $0.4 \mathrm{~mm}$. The final structures in ABS, due to the construction limit imposed by the platform size of the A8 machine, which has a maximum area of $20 \times 20 \mathrm{~cm}^{2}$, are composed of a group of 8 unit cells of $2 \times 2 \mathrm{~cm}^{2}$, with a total area of $16 \times 16 \mathrm{~cm}^{2}$ with a thickness of $1.6 \mathrm{~mm}$. Fig. 9 illustrates the four prototypes built with this process.

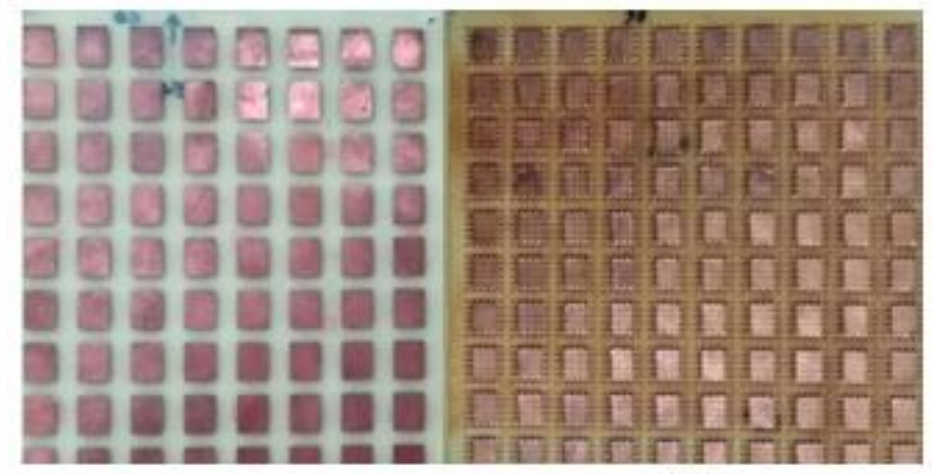

(a)

(b)

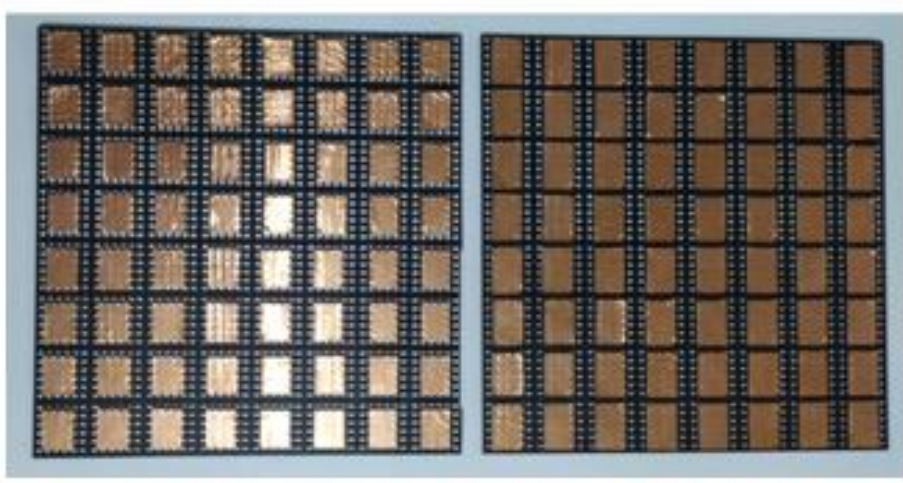

(c)

(d)

Fig. 9. Built prototypes: (a) FSS without EBG, (b) FSS with cylindrical holes EBG, (c) FSS with square holes EBG, and (d) FSS with square holes EBG and modified patch.

The frequency response of the prototypes was measured using a two-port Agilent E5071C network vector analyzer, SAS-571 rigid double horn antennas, and a measurement window. Unobstructed window measurements were taken, referred to as free space measurement and four with built prototypes. Fig. 10 illustrates the measurement setup.

The first prototype measured was the FSS with a rectangular patch with periodicity $P=20 \mathrm{~mm}$, width $W=12 \mathrm{~mm}$, length $L=15 \mathrm{~mm}$ without EBG. Fig. 11 illustrates the comparison between the simulated results obtained with the ANSYS HFSS software and the measured results. Simulated results show a resonance at $9.24 \mathrm{GHz}$ and a grating lobe at $13.02 \mathrm{GHz}$ and the measured values show 
a resonance at $8.84 \mathrm{GHz}$ and a grating lobe at $13.28 \mathrm{GHz}$. The differences were $4,3 \%$ for the resonance frequency and $2 \%$ for the grating lobe, what is a good agreement.

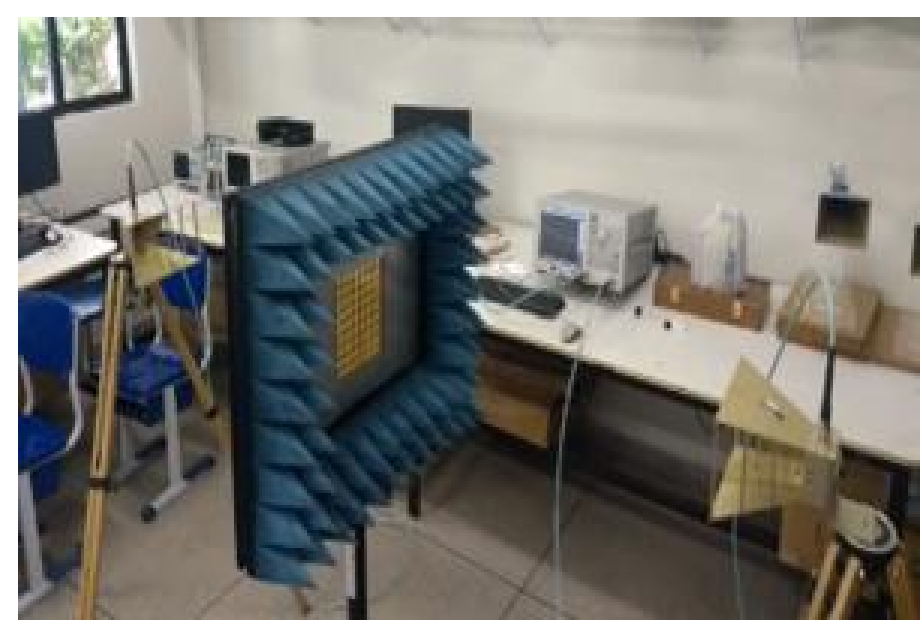

Fig. 10. Measurement setup.

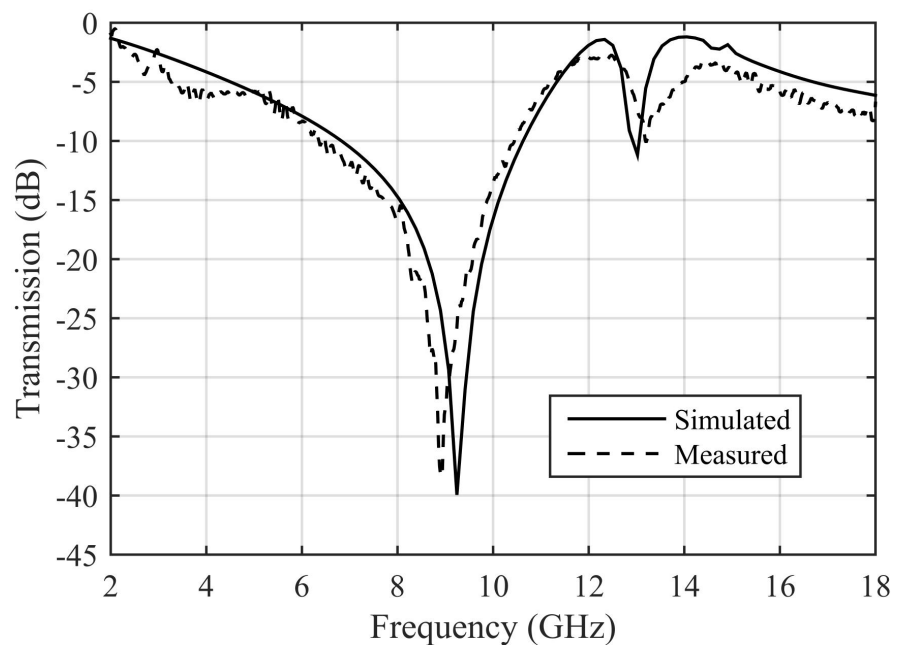

Fig. 11. Comparison between simulated and measured results of the first prototype.

The second prototype measured was the FSS integrated with an EBG with cylindrical holes for the same physical dimensions of the prototype of Fig. 11. The EBG has cylindrical holes with $3 \mathrm{~mm}$ of diameter and a pitch of $3.3 \mathrm{~mm}$. Fig. 12 illustrates the comparison between simulated and measured results. Simulated results show a resonance at $10.07 \mathrm{GHz}$ and the grating lobe suffered an attenuation of $6 \mathrm{~dB}$, whereas the measured results show a resonance at $9.94 \mathrm{GHz}$ and the grating lobe was attenuated by $4 \mathrm{~dB}$. The holes produced a decrease in effective permittivity and, consequently, the resonance frequency increases. We can observe the attenuation of the grating lobe and a difference between results of $1.29 \%$ in terms of the resonance frequency. Again, a good agreement between the results was observed.

So, to reduce the computational effort, we proposed a dielectric with an EBG with square holes. The idea is to use a 3D printer to build a substrate with an EBG with square holes and reduce the simulation time. The material used was the acrylonitrile-butadiene-styrene (ABS) and the built dielectric has $1.6 \mathrm{~mm}$ of thickness and a relative permittivity of 3.3. This can be useful for 
optimization processes. The third prototype measured was the FSS with an EBG with square holes for the same physical dimensions of the unit cell used in the first prototype. The EBG has square holes of $2.5 \mathrm{~mm}$ of side and a pitch of $3.3 \mathrm{~mm}$. Fig. 13 illustrates the comparison between simulated and measured results. Simulated results show a resonance at $11.86 \mathrm{GHz}$ and the grating lobe was attenuated by $6 \mathrm{~dB}$, whereas the measured results show a resonance at $11.94 \mathrm{GHz}$ and the grating lobe was attenuated by $6 \mathrm{~dB}$ as well. We can observe a difference between results of $0.67 \%$ in terms of resonance frequency. In addition, we observed a second lobe with $-20 \mathrm{~dB}$ of level at $10.68 \mathrm{GHz}$. Due to the fact that our patches are handmade, we noticed several patches with bigger dimensions than they should have, which caused a second resonance to appear at a lower frequency. However, as it is an FSS stopband, this is not a problem, because what matters is that in the entire operation frequency band, the rejection level is greater than $10 \mathrm{~dB}$, which is what happens.

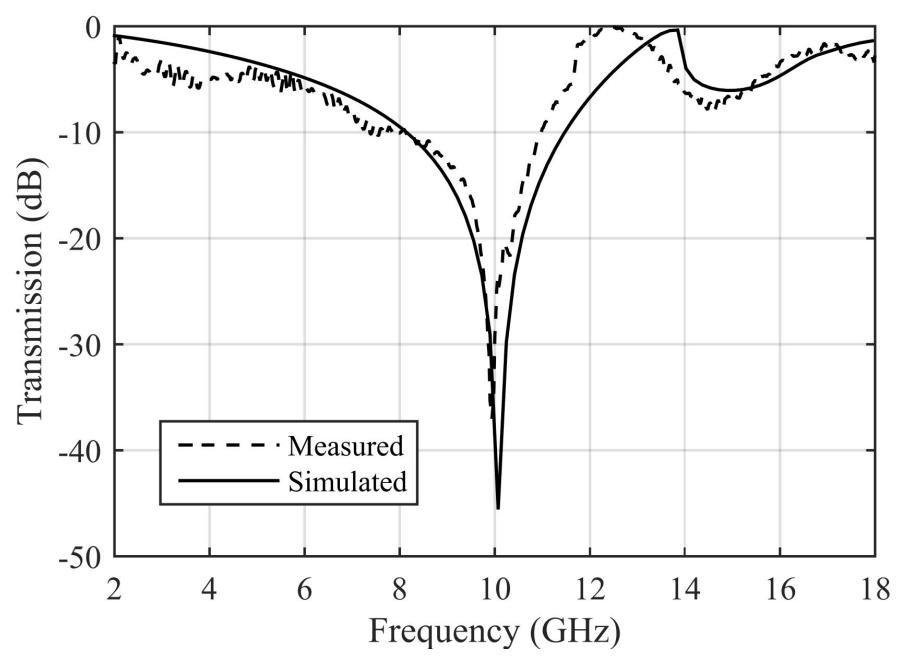

Fig. 12. Comparison between simulated and measured results of the second prototype.

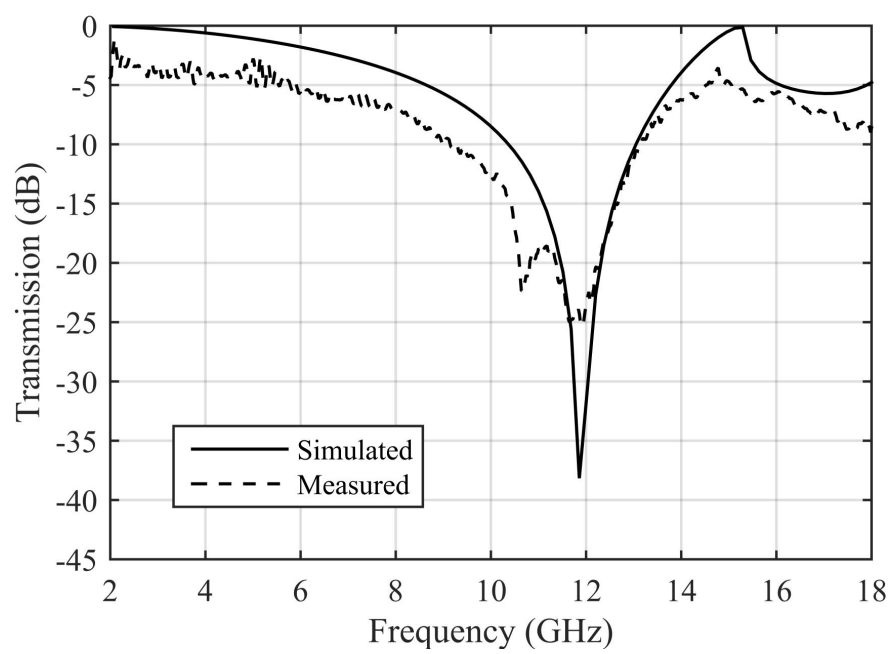

Fig. 13. Comparison between simulated and measured results of the third prototype. 
We resized the patch of the unit cell, trying to bring the resonance frequency near to the original value, 9.24 GHz. So, we proposed a fourth prototype (Fig. 7(d)) with a dielectric with square holes' EBG. We maintained the values of $P$ and $W$, and increased the length $L$ for $18.5 \mathrm{~mm}$. The EBG has square holes of $2.5 \mathrm{~mm}$ of side and a pitch of $3.3 \mathrm{~mm}$. Fig. 14 illustrates the comparison between simulated and measured results. Simulated results show a resonance at $10.22 \mathrm{GHz}$ and the measured results show a resonance at $10.04 \mathrm{GHz}$. In both cases, the attenuation of the grating lobe was $5 \mathrm{~dB} \mathrm{We}$ can observe a difference between results of $1.76 \%$ in terms of resonance frequency. In addition, we observed a second lobe with $-20 \mathrm{~dB}$ of level at $10.94 \mathrm{GHz}$. Due to the fact that our patches are handmade, we noticed several patches with smaller dimensions than they should have, which caused a second resonance to appear at a higher frequency. Again, as it is an FSS stopband, this is not a problem, because what matters is that in the entire operation frequency band, the rejection level is greater than $10 \mathrm{~dB}$, which is what happens.

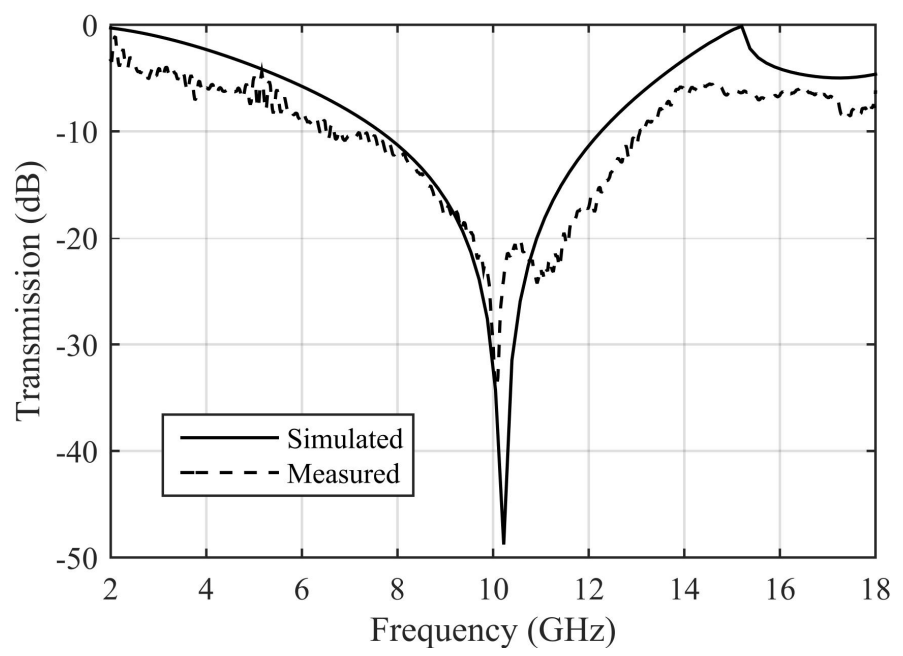

Fig. 14. Comparison between simulated and measured results of the fourth prototype.

\section{CONCLUSIONS}

The aim of this study was to propose and validate, through computer simulations and prototype measurements, the use of EBG for suppressing lobes in FSS. The Brillouin diagram was obtained for two structures to show how the EBG suppressed higher order modes. The measured results showed a good agreement with the simulated results obtained with the commercial software ANSYS HFSS. An alternative type of EBG with square holes was proposed to reduce the computational effort in simulations. Four different FSS were used to show the suppression of the grating lobes. Prototypes were built and measured. The measured frequency responses presented suppression of an order of 6 $\mathrm{dB}$ or more. In this work, it was proven that the signal confinement due to the EBG application, allows a new functionality for planar structures when used as FSS, which is the suppression of spurious modes provoked by stimulated scattering of the signal within of the structure. The FSS with EBG with square holes allows a simulation time $70 \%$ lower than FSS with cylindrical holes EBG, showing that EBG with square holes is more appropriated for the optimization process. The handmade 
process to fabricated the metallic patches produces differences between simulated and measured results for FSS with EBG, but the differences were less than $5 \%$. However, it is observed that the manufacturing process would be industrial and not handmade, if some practical application is aimed. In addition, we observed a second lobe with $-20 \mathrm{~dB}$ of level. Due to the fact that our patches are handmade, we noticed several patches with smaller or bigger dimensions than they should have (depends on the prototype), which caused a second resonance to appear at a higher or lower frequency. However, as it is an FSS stopband, this is not a problem, because what matters is that in the entire operation frequency band, the rejection level is greater than $10 \mathrm{~dB}$, which is what happens.

\section{REFERENCES}

[1] D. M. Pozar, Microwave Engineering, NY, USA, 2011.

[2] J. S. Hong and M. J. Lancaster, Microstrip Filters for RF/Microwave Applications, NJ, USA, John Wiley \& Sons, Inc. 2001.

[3] J. Tang, D. Deslandes, K. Wu, "Spurious mode suppressing technique for performance enhancement of hybrid planar/NRD-guide circuits", 2000 Asia-Pacific Microwave Conference, Australia, Sidney, December, 2000.

[4] X. Yin, H. Zhang, X. Y. Huang, and H. Y. Xu, "Spurious Modes Reduction in a Patch Antenna Using an ENG-Based Microstrip Transmission Line Filter", Progress In Electromagnetics Research C, 2012, 25, pp. 41 - 54.

[5] I. F. Costa, A. L. P. S. Campos, A. Gomes Neto, "Sharp Rejection and Wide Stopband Microstrip Lowpass Filters using Complementary Split Ring Resonators". Journal of Microwaves, Optoelectronics and Electromagnetic Applications, vol.17, pp $136-145,2018$.

[6] R. Mittra, C. H. Chan, and T. Cwik, "Techniques for analyzing frequency selective surfaces: A review", Proceedings of the IEEE, vol. 76, no. 12, pp. 1593-1616, 1988.

[7] J. Acharjee et al., "Suppressing up to fourth harmonic of an ISM band microstrip patch antenna using compact defected ground structures", Microwave and Optical Technology Letters, vol. 59, no. 9, pp 2254-2259, 2017.

[8] F. C. B. Sena and J. P. Silva, "Harmonic suppression using optimised hexagonal defected ground structure by genetic algorithm", IET Microwaves, Antennas \& Propagation, vol. 12, no. 10, pp 1645-1648, 2018.

[9] S. S. Karthikeyan, and R. S. Kshetrimayum, "Compact, Deep and Wide Rejection Bandwidth Low-pass Filter Using Open Complementary Split Ring Resonator", Microwave and Optical Technology Letters, vol. 53, no. 4, pp. 845-848, 2011.

[10] R. Y. Yang, et al., "Design of a Compact and Sharp Rejection Lowpass Filter with a Wide Stopband", Journal of Electromagnetic Waves Applications, vol. 26, no. 17-18, pp. 2284-2290, 2012.

[11] L. F. Shi, Z. M. Sun, G. X. Liu, and S. Chen, "Hybrid-Embedded EBG Structure for Ultrawideband Suppression of SSN", IEEE Transactions on Electromagnetic Compatibility, vol. 60, no. 3, pp 747 - 753, 2018.

[12] A. O. Guimarães, J. P. da Silva, J. P. Pereira, "Analysis of a microstrip antenna with variation on substrate PBG hexagonal", Microwave and Optical Technology Letters, vol. 58, no. 4, pp. 826-831, 2016.

[13] B. O. de Andrade and L. M. de Mendonca, "Frequency invariance, gain improvement, and fast design in 3D-printed photonic band gap antennas with square holes". Microwave and Optical Technology Letters, vol. 61, pp. 2295-2305, 2019.

[14] N. Ripin, R. A. Awang, A. A. Sulaiman, N. H. Baba, and S. Subahir, "Rectangular microstrip patch antenna with EBG structure", 2012 IEEE Student Conference on Research and Development (SCOReD) , Malaysia, Pulau Pinang, December, 2012

[15] P. Chen, Y. Ru, and L. K. Liao, "A novel planar electromagnetic band-gap structure for SSN suppression”, Journal of Electronic Infrared Technology, vol. 36, no. 11, pp. 2775-2780, 2014.

[16] A. L. P. S. Campos, T. L. Silva, and A. Gomes Neto, "Multiband Frequency Selective Surfaces with Simple Modification of a Rectangular Patch Element", Microwave and Optical Technology Letters, vol. 55, pp. 2943-2946, 2013.

[17] N. Jaglan and S. D. Gupta, "Design and Analysis of Performance Enhanced Microstrip Patch Antenna with EBG Substrate", International Journal of Microwave and Optical Technology, vol.10, no. 2, pp. 79 - 88, 2015.

[18] B. M. Iravani and O. M. Ramahi, "On the Suppression Band and Bandgap of Planar Electromagnetic Bandgap Structures”, International Journal of Antennas and Propagation, vol. 2014, pp. 1 - 11, 2014. 